$\Rightarrow$ CELL SIGNALLING

\title{
STINGing revelations
}

The cGAS (cyclic-GMP-AMP (stimulator of interferon genes) pathway detects pathogenic DNA (bacterial or viral) and cytosolic endogenous DNA (resulting from DNA damage), and induces innate immune responses - mostly the production of interferon through the activation of TANK binding kinase 1 (TBK1). These responses support clearance of pathogens, but may also lead to autoinflammatory diseases. Four studies from Nature and Cell provide new insights into the mechanism, functions and regulation of the cGAS-STING pathway.

STING is a transmembrane homodimer that resides in the endoplasmic reticulum (ER). Activation of STING by cGAMP involves its multimerization, trafficking towards the Golgi and TBK1 activation, but how STINGmediated signalling occurs at the molecular level remains poorly understood. In back-to-back reports, Shang et al. and Zhang et al. investigated conformational changes associated with STING activation by cryo-EM. Shang et al. found that upon cGAMP binding, the ligandbinding domain (LBD) of STING rotates by $180^{\circ}$ with respect to the transmembrane domain (TMD), which causes loss of intersubunit crossover at the connecting regions between the TMDs and the LBDs of the two dimer subunits. Various mutations associated with autoinflammatory diseases localize to this connecting region and may promote $\mathrm{LBD}$ rotation to induce cGAMP-independent STING signalling. Furthermore, a loop region between the second and third helices

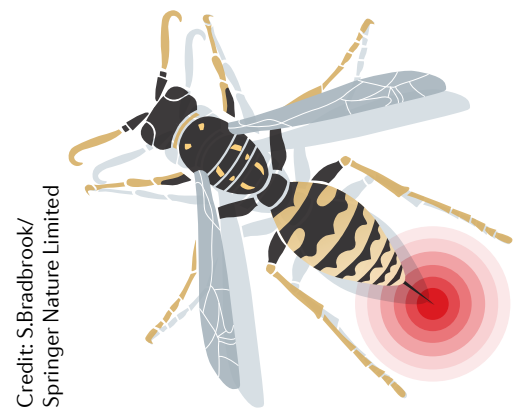

of the LBD was determined to be an interface for STING multimerization, and the movement of these two helices - which is likely coupled to conformational changes in the TMD-LBD connecting region - was associated with STING activation.

Zhang et al. defined a molecular interface for STING-TBK1 interaction and demonstrated that TBK1 binds to STING even in its cGAMP-unbound state. Importantly, the STING-TBK1 interaction was strengthened by STING activation, and downstream signalling was sensitive to the disruption of STING multimerization. Thus, by bringing multiple TBK1 molecules into close proximity, multimerization of STING likely supports TBK1 trans-autophosphorylation and activation.

Gui et al. found that in addition to TBK1 activation, cytosolic DNA also induces lipidation of light chain 3 (LC3), which drives the expansion of autophagosome membranes during autophagy. cGAMP delivery into cells was sufficient to increase LC3 lipidation, which occurred independently of canonical autophagy inducers ULK kinases and Belin-1, suggesting a new role of STING in non-canonical autophagy. Trafficking of activated STING to the ER-Golgi intermediate compartment (ERGIC) was required for CGAMP-induced LC3 lipidation, and ERGIC membranes were found to be a key source of autophagosomal membranes during cGAMP-induced autophagy. Stimulation of cells with cGAMP augmented clearance of cytoplasmic DNA associated with DNA damage or viral infection. It also limited viral titres in a manner dependent on functional LC3 lipidation. This newly discovered role of active STING in autophagic clearance of cytoplasmic DNA is likely a primordial function of the cGAS-STING pathway, as in certain species, including Nematostella vectensis (starlet sea anemone) and Xenopus laevis, STING lacks the TBK1-binding region and is unable to induce inflammatory responses but is still functional in LC3 lipidation.

PtdIns $(4,5) \mathrm{P}_{2}-$
mediated
binding of
cGAS to
autophagic
membranes
could enable
activation of
STING in the
context of
autophagy

Barnett et al. showed that in macrophages, cGAS localizes primarily to the plasma membrane - apart from a smaller pool in the nucleus - thereby contradicting the prevailing view that cGAS is cytosolic. Plasma-membrane targeting was found to be mediated by the recognition of phosphatidylinositol4,5-bisphosphate $\left(\operatorname{PtdIns}(4,5) \mathrm{P}_{2}\right)$ by the cGAS N-terminal domain. Nevertheless, cGAS was released from the plasma membrane in the presence of cytosolic DNA and this release was required for downstream immunogenic signalling. The localization of cGAS to the plasma membrane was associated with lower production of interferon in the presence of cytosolic endogenous DNA and with increased cell survival in response to genotoxic insults. At the same time, cGAS that was unable to bind to the plasma membrane was less efficient at raising immune response to viral infection. Thus, the distribution of cGAS between the plasma membrane and the cytosol determines cellular responses to pathogenic versus endogenous DNA. Interestingly, the degree of cGAS targeting to the plasma membrane varied between cell types - non-phagocytic cells had larger cytosolic pools than phagocytic cells - indicating context-dependent regulation of cGAS subcellular distribution. In addition, because PtdIns $(4,5) \mathrm{P}_{2}$ also regulates autophagic-membrane trafficking, PtdIns $(4,5) \mathrm{P}_{2}$-mediated binding of cGAS to autophagic membranes could enable activation of STING during autophagy, as shown by Gui et al.

The increasing understanding of the cGAS-STING pathway raises possibilities for precise regulation of cellular responses to cytosolic DNA to selectively enhance pathogen clearance and limit toxicity associated with the recognition of endogenous DNA.

Paulina Strzyz 